\title{
Characteristics of Jupiter's X-ray auroral hot spot emissions using Chandra
}

\author{
D. M. Weigt ${ }^{1}$, C. M. Jackman ${ }^{2}$, M. F. Vogt ${ }^{3}$, H. Manners ${ }^{4}$, W. R. Dunn ${ }^{5}$, G. \\ R. Gladstone ${ }^{6,7}$, R. Kraft ${ }^{8}$, G. Branduardi-Raymont ${ }^{5}$, C. K. Louis ${ }^{2}$, S. C. \\ McEntee ${ }^{2,9}$ \\ ${ }^{1}$ School of Physics and Astronomy, University of Southampton, Southampton, UK \\ ${ }^{2}$ School of Cosmic Physics, DIAS Dunsink Observatory, Dublin Institute for Advanced Studies, Dublin 15, \\ Ireland \\ ${ }^{3}$ Center for Space Physics, Boston University, Boston, MA, USA \\ ${ }^{4}$ Mullard Space Science Laboratory, Department of Space and Climate Physics, University College \\ London, Dorking, UK \\ ${ }^{5}$ The Centre for Planetary Science at UCL/Birkbeck, London, UK \\ ${ }^{6}$ Space Science and Engineering Division, Southwest Research Institute, San Antonio, TX, USA \\ ${ }^{7}$ Department of Physics and Astronomy, University of Texas at San Antonio, San Antonio, TX, USA \\ ${ }^{8}$ Harvard-Smithsonian Center for Astrophysics, Smithsonian Astrophysical Observatory, Cambridge, MA, \\ USA \\ ${ }^{9}$ School of Physics, Trinity College Dublin, Dublin 12, Ireland
}

\section{Key Points:}

- We present the first statistical study looking at the behaviour of Jupiter's northern X-ray auroral hot spot from 20 years of Chandra data.

- The X-rays map close to the magnetopause from noon to dusk, with the center of the averaged hot spot emissions mapping to noon.

- Our analysis suggests that the X-ray driver(s) may be linked with ultra-low frequency wave activity along the magnetopause.

Corresponding author: Dale Weigt, D.M.Weigt@soton.ac.uk 


\begin{abstract}
To help understand and determine the driver of jovian auroral X-rays, we present the first statistical study to focus on the morphology and dynamics of the jovian northern hot spot (NHS) using Chandra data. The catalogue we explore dates from 18 December 2000 up to and including 8 September 2019. Using a numerical criterion, we characterize the typical and extreme behaviour of the concentrated NHS emissions across the catalogue. The mean power of the NHS is found to be $1.91 \mathrm{GW}$ with a maximum brightness of 2.02 Rayleighs (R), representing by far the brightest parts of the jovian X-ray spectrum. We report a statistically significant region of emissions at the NHS center which is always present, the averaged hot spot nucleus (AHSNuc), with mean power of $0.57 \mathrm{GW}$ and inferred average brightness of $\sim 1.2 \mathrm{R}$. We use a flux equivalence mapping model to link this distinct region of X-ray output to a likely source location and find that the majority of mappable NHS photons emanate from the pre-dusk to pre-midnight sector, coincident with the dusk flank boundary. A smaller cluster maps to the noon magnetopause boundary, dominated by the AHSNuc, suggesting that there may be multiple drivers of X-ray emissions. On application of timing analysis techniques (Rayleigh, Monte Carlo, Jackknife), we identify several instances of statistically significant quasi-periodic oscillations (QPOs) in the NHS photons ranging from 2.3-min to 36.4-min, suggesting possible links with ultra-low frequency activity on the magnetopause boundary (e.g. dayside reconnection, Kelvin-Helmholtz instabilities).
\end{abstract}

\title{
Plain Language Summary
}

The auroral emissions (northern and southern lights) on Jupiter are the most powerful in our Solar System and have been observed across the electromagnetic spectrum. The cause, or driver, of Jupiter's auroras is still an open question with lots of scientific debate. The solar wind can have an effect, as can Jupiter's volcanic moon Io. The plasma and magnetic field interactions can produce auroras on Jupiter in the X-ray waveband. These powerful X-ray emissions can be observed by telescopes like the Chandra X-ray Observatory (CXO) that orbit Earth. The X-ray data we analyze here have been found to flash or pulsate at certain periods, spanning the $\sim 20$ years Chandra has observed Jupiter. We use mapping and timing analysis techniques to analyze the entire catalogue from the high-resolution camera on-board Chandra. We report significant auroral X-ray regions and pulsations in the north to help us provide an answer for the possible multiple X-ray drivers.

\section{Introduction}

Jupiter has strong auroral X-ray emissions which are observed to be concentrated into a "hot spot". The first spatially resolved X-ray auroral "hot spot" was observed by Chandra $\sim 20$ years ago, discovered by Gladstone et al. (2002) in the northern polar region. The term "hot spot" was coined to define the region where most of the X-ray emissions were found from the Chandra observation. Gladstone et al. (2002) defined the hot spot region as a $5^{\circ}$ radius circle centered on $170^{\circ}$ System III (S3) longitude and $65^{\circ}$ latitude. The origin of the ions producing the X-ray emissions were shown to have their source in the outer magnetosphere, $>30$ Jupiter radii $\left(\mathrm{R}_{J}\right)$ from the planet. Timing analysis of the 113 photons within the hot spot showed a flaring of X-ray emissions or quasi-periodic oscillation $(\mathrm{QPO})$ at $\sim 45 \mathrm{~min}$, similar to pulsations found in the radio emission from the Ulysses flyby (MacDowall et al., 1993) and electron bursts from the Cassini flyby (Krimigis et al., 2002).

Since then, subsequent Chandra and X-ray Multi-Mirror Mission (XMM-Newton) (Jansen et al., 2001) observations have allowed us to analyze the morphology and composition of the hot spot emissions in more detail at both poles. We now know that the hot spot consists of soft X-rays (SXRs, energies $<2 \mathrm{keV}$ ) (Branduardi-Raymont et al., 
2008) observed at high latitudes, exhibiting a large range of QPOs (Dunn et al., 2016, 2017; Elsner et al., 2005; Gladstone et al., 2002; Jackman et al., 2018; Kimura et al., 2016; Weigt et al., 2020; Wibisono et al., 2020) which may be correlated with emissions in other wavebands (Dunn et al., 2020a). These SXRs are thought to be produced by charge exchange between ions precipitating down into the jovian atmosphere and the neutrals that reside there (Bhardwaj \& Gladstone, 2000; Cravens et al., 1995). This heavy ion precipitation can originate from either the open field lines in the magnetosphere connected to the solar wind or on the closed field lines that map to the outer regions of the magnetosphere (Cravens et al., 2003). Energetic heavy ions are found to be the main source of the total X-ray power output (1 GW to a few GWs) (Houston et al., 2020) from the most recent models and in-situ Juno data (Bolton et al., 2017). The X-ray auroral spectrum is well-fit by atomic charge exchange spectral lines, with the spectrum typically best fit by an iogenic population of sulfur (S) and oxygen (O) (Elsner et al., 2005; BranduardiRaymont et al., 2007; Hui et al., 2010; Ozak et al., 2010, 2013; Houston et al., 2020; Dunn et al., 2020b). However, alongside $\mathrm{S}$ and $\mathrm{O}$, there are individual observations in which the addition of charge exchange lines from solar wind ions colliding with the atmosphere can improve the spectral fit (Branduardi-Raymont et al., 2007; Hui et al., 2010; Dunn et al., 2020b). In order for this process to operate within the jovian magnetosphere, fieldaligned electric fields capable of producing very high potentials $(\sim 0.2-8 \mathrm{MV})$ are needed between the ionosphere and magnetosphere (Cravens et al., 2003; Bunce et al., 2004). Such high potentials were observed at Jupiter's poles by the Jupiter Energetic Particle Detector Instrument (JEDI) (Mauk et al., 2017) on-board Juno. The MV potentials were associated with charge stripping of heavy iogenic ions required for SXR production (Clark et al., 2020). This combination of remote sensing data from the X-ray telescopes and other wavebands with available in situ probe data is vital to enhance our understanding of the jovian X-ray emissions. The in situ data provides us with the magnetospheric conditions during the observation window, giving the X-ray observations context and determining a possible shared driver across all observed emissions.

The first spatially resolved observation of the southern hot spot was reported by Branduardi-Raymont et al. (2008). Dunn et al. (2017) studied both the northern and southern hot spots for the first time, during an observation when the tilt of the planet was favourable for both poles to be observed. During this observation, the northern and southern hot spots were non-conjugate and found to pulsate at different quasi-periods with a significant 9-11 min QPO in the South and no clear significant pulsations in the North. This suggests that the driver for both hot spots may be different or the same driver was triggered independently in order to produce the different temporal behaviour in the QPOs observed. This independent nature between the hot spots was also found by Weigt et al. (2020). Two significant QPOs were found in the North (lasting for less than one Jupiter rotation) but none in the South, during a $~ 10$-hr Chandra observation (18 June 2017) during Juno apojove (AJ) 6. The magnetosphere was inferred to be compressed during this time from the Jovian Auroral Distributions Experiment (JADE) (McComas et al., 2017) and the Jupiter Energetic Particle Detector (JEDI) (Mauk et al., 2017) onboard Juno. From a concurrent $\sim 24$-hr XMM-Newton observation (in which the beginning of the interval overlapped with the final 5 hours of the Chandra campaign), Wibisono et al. (2020) found non-conjugate behaviour simultaneously with Chandra and observed the same significant QPO in the North (26-28 min). However outside of the Chandra window, both the northern and southern auroral regions pulsated with a 23-to 27-min periodicity for $\sim 12.5$ hours (more than one Jupiter rotation). This suggests that the nonconjugate behaviour of the North and South arises from different drivers producing similar QPOs or as a result from the same driver producing a lag in the emissions we observe (with changing phase). It is apparent from the June 2017 campaigns alone that the emissions from both hot spot emissions are highly variable over a short timescale, raising further questions about the possible drivers capable of producing such pulsed emissions. 
In order to determine how variable the hot spot temporal and spatial behaviour is, we analyze the full Chandra catalogue in a statistical study. This will allow the typical and extreme behaviours of the hot spot emissions to be studied in more detail. Finding these types of behaviour will allow us to have a better grasp of how the X-rays change with different magnetospheric conditions (e.g. solar wind, Io activity) which can be explored in detail in the future. We apply the algorithm and definitions used by Weigt et al. (2020) to find significance in the "average" hot spot morphology (i.e. the occurrence of X-ray emissions within the hot spot across all observations) and where the emission maps to using a flux equivalence mapping model (Vogt et al., 2011, 2015). To ensure our interpretations of the mapping are correct, we explore the limitations and sensitivity of the model to possible uncertainties such as the ionospheric position (in jovian S3 longitude and latitude coordinates) of the photons detected. From the timing analysis, we create a catalogue of results which can be compared to previous statistical studies looking into the temporal behaviour of the auroral hot spot (such as Jackman et al. (2018)) and allow us to explore the possible spatial dependence of the QPOs (i.e. are the significant pulsations only found in a particular region of the hot spot?). This allows us to check the validity and robustness of our timing analysis as well as comparing any significant QPOs found here to other studies.

In section 2, we discuss the Chandra catalogue used in our statistical study and the techniques used to process this large data set. Section 3 discusses the average morphology and the statistical significance of the hot spot emissions. The hot spot emissions are then mapped using the Vogt et al. (2011, 2015) method to find the most likely location of the driver, considering possible uncertainties that may have an effect on our interpretations. Furthermore, we perform timing analysis on the full Chandra catalogue to find and confirm any significant QPOs and explore their possible spatial dependence. Section 4 contains a detailed discussion of our results from the statistical study and our interpretation of the behaviours observed from the hot spot emissions.

\section{Dataset}

The data used in this statistical study were obtained by the high-resolution camera (HRC-I) on board the Chandra X-ray observatory (Weisskopf et al., 2000). The Chandra HRC-I consists of a single large-format microchannel plate which provides high spatial resolution of $\sim 0.4$ arcsec over a $30 \operatorname{arcmin} \times 30$ arcmin field of view. The best image quality is found at the center of the field of view, where the aim point of the camera is located. Chandra HRC-I can record X-ray photons with energy in the range 0.08 - $10 \mathrm{keV}$. The HRC-I typically observes an average count rate of $\sim 0.035$ counts/s (with $\sim 0.7$ counts/s maximum) from the typically more intense northern auroral X-ray emissions. The instrument has maximum sensitivity to the lower energy pulsed emissions from the SXRs which allows us to identify clearly the longitude and latitude of the X-ray timetagged photons, with a spatial resolution of $1^{\circ} \mathrm{S} 3$ longitude $\times 1^{\circ}$ latitude (after processing).

The Chandra HRC-I data span $\sim 20$ years with 29 observations in total (to date including the Juno era) from 18 December 2000. As shown in Table S1, 8 observations spread over several campaigns to coincide with flybys of spacecraft close to Jupiter, or to the expected arrival of a coronal mass ejection (CME). This is augmented by 21 observations since 2016 spanning the approach phase and early orbits of the Juno spacecraft. Many of the Chandra campaigns were also carried out in tandem with other remote sensing observatories (across multiple wavelengths). We only focus on the Chandra observations in this study which span almost two full solar cycles. All the observation dates with the duration, concurrent missions during the Chandra interval and visibility of auroral regions are shown in Table S1 in the Supplementary Information, allowing for future comparative studies. We define the northern auroral region as poleward of $40^{\circ}$ latitude with an S3 longitude of $100^{\circ}$ to $240^{\circ}$. The southern auroral region we de- 
fine as poleward of $-60^{\circ}$ latitude poleward with no longitude constraint as the hot spot emissions are more diffuse and are located near the South pole. This therefore makes it difficult to find the location of the most intense southern emissions.

With the high spatial resolution of Chandra HRC-I, the X-ray emission can be mapped onto the jovian disk using 2-D histograms. This is carried out by using the Gaussian point spread function (PSF) of the instrument, with a full-width at half maximum (FWHM) of 0.4 arcsec, transformed into S3 coordinates. This high spatial resolution allows the position and morphology of specific features within the X-ray emissions, such as the hot spot, to be spatially down-selected and studied in greater detail. Prior to mapping the X-ray emissions, we need to correct for the planet's motion as it moves across the detector. Both the data correction and mapping processes are carried out using a Python pipeline which assumes the X-ray emissions occur at an altitude of $400 \mathrm{~km}$ above the 1bar atmosphere. The PSF size of the HRC is assumed to be 25 arcsec with a FWHM of 0.8 arcsec, at variance with the FWHM of the instrument. Further details about the Python pipeline can be found in Weigt et al. (2020).

With polar projected 2-D histograms mapping X-ray brightness onto Jupiter's surface, we can observe the traversal of the hot spot across the disk as Chandra HRC-I observes Jupiter. The hot spot traverses the disk for $\sim 5$ hours, and while most of the observations from the Chandra catalogue are $\sim 10$ hours in duration, some are shorter and have been optimized for hot spot viewing. From these observations, 28 out of 29 were useable and this is the catalogue we analyze in detail here using our mapping algorithm. The observation that could not be mapped properly, ObsID 18303 in Table S1, was unable to be accurately mapped. This resulted from Jupiter being off-center on the detector. This misalignment on the detector therefore inhibits optimal mapping of this observation as the PSF increases with distance away from the center of the detector. This can lead to greater uncertainties when mapping the emissions.

\section{Results}

The specific structures within the X-ray aurora can be studied in more detail by defining select spatial regions within the X-ray emissions and analyzing their temporal behaviour. Dunn et al. (2020a) recently found that the soft X-ray aurora can be separated into three different sub-categories: regularly pulsed emission, irregularly pulsed emission and flickering aurora. The pulsed behaviours were found to be associated with Xrays flaring during short-lived $(\sim 1-2 \mathrm{~min})$, concentrated intervals which are followed immediately with longer intervals of dim to no X-ray emissions. The 'flickering' behaviour of the soft X-ray aurora was observed to vary in brightness over short time scales (1-2 min) but remained continuous throughout the observation (i.e. no extended intervals devoid of X-rays emission). In this study, we will focus on the former two types of X-ray aurora where the more intense SXRs are found to be concentrated in a hot spot region. We analyze in detail the variable spatial and temporal behaviour of these emissions located within this region using a variety of techniques.

\subsection{Overall morphological characteristics of the X-ray emissions}

With the large catalogue of Chandra HRC-I observations now available, it is now possible to explore both the average and extreme conditions of jovian X-ray emissions. In this study, we begin by examining planetographic polar projected 2-D histograms of the brightness of all auroral X-rays in the catalogue. The polar plots of the averaged Xray emission across the majority of the catalogue (28 observations) are shown in Figure 1. The average X-ray emissions were found by mapping all photons in the catalogue to their ionospheric positions (S3 longitude, latitude). At each position, the flux found in each $1^{\circ} \mathrm{S} 3$ longitude $\times 1^{\circ}$ latitude bin (the typical spatial resolution of our data) was averaged over the catalogue, with a typical observation time of $\sim 10.2$ hours for both the North 



Figure 1. Planetographic polar plots of the average X-ray emission as viewed from above (a) Jupiter's north and (b) south poles from the 28 out of 29 observations of the Chandra HRC-I catalogue. The azimuth angle (in joviographic longitude) within the polar plot (in degrees) is indicated around the plot. The concentric circles represent $10^{\circ}$ latitude increments with latitudes $\geq$ $\left|40^{\circ}\right|$ highlighted. The brightness of the X-ray emissions is proportional to the photon flux, calculated from the average point spread function (PSF) across all 29 observations. This is denoted by the color bar below in units of Rayleighs (R). The PSF shows the number density of photons detected with an uncertainty on their position (spreading of the PSF). The regions which have little to no X-ray emissions are represented in white. The Voyager Io Pioneer 4 (VIP4) (Connerney et al., 1998) Io and Grodent Anomaly Model (GAM) (Grodent et al., 2008) Ganymede footprints are plotted in (a) and the VIP4 Io and Ganymede footprints in (b). The footprints in both panels are given by the dashed and solid black lines respectively.

and South auroral regions. Such 2-D histograms allow the overall morphology, position and properties of the hot spot emissions to be analyzed in greater detail than just the photon data alone. Figure 1 shows the X-ray emissions as viewed from above (a) the north and (b) south pole. The Grodent Anomaly Model (GAM) (Grodent et al., 2008) Ganymede footprint in the North pole is plotted in panel (a). The Voyager Io Pioneer 4 (VIP4) (Connerney et al., 1998) Io footprint is plotted in both panels and the VIP4 Ganymede footprint in panel (b). These contours are used in all figures herein for the North and South poles and allow us to provide context to the position of the emissions on the poles and where they map to magnetically in the magnetosphere.

Figure 1 shows a clear asymmetry in the brightness between the North and South hot spot (herein referred to as NHS and SHS respectively), as represented by the color bar. As depicted in Figure 1a), the most intense NHS emission is located in a tear-drop shape with more diffuse emission (dark blue) surrounding the region, extending almost out to the pole at $\mathrm{S} 3$ longitude of $0^{\circ}$. The more diffuse emissions are located between longitudes of $\sim 90^{\circ}$ to $225^{\circ}$ and are more widespread than the most intense NHS emissions. The X-rays here are observed to be spread poleward of the Ganymede footprint (solid) and extend to the Io footprint (dashed) and beyond in regions closer to $225^{\circ}$. 




Figure 2. Histograms of the properties of the X-ray aurora from the Chandra observation catalogue. Top panels (a) - (c) show values for the North, and bottom panels (d) - (f) show values for the South. First column (panels (a) and (d)) show the power of the X-ray aurora. The following columns show the energy flux ((b) and (e)) and maximum brightness ((c) and (f)) as observed from Chandra (i.e. at Earth). The mean, $\mu$, median, $M$, and standard deviation, $\sigma$, of the distributions are shown in each histogram. The mean and median of each distribution are denoted by the solid and and dashed vertical lines respectively.

The SHS is observed to be far more diffuse with the most intense emissions located within the Ganymede footprint (Figure 1b)). The asymmetry may be a result of unfavourable viewing geometry of the SHS throughout the catalogue (Dunn et al., 2017). However, in our study, we find this asymmetric auroral behaviour throughout each of the observations, including the 12 observations which had equal viewing of both auroral regions (Table S1). Therefore viewing geometry may contribute to the non-conjugate behaviour but will not be the most dominant effect. The more prominent mechanisms that may contribute to the asymmetry may result from the very different magnetic field strengths and topologies between both poles (Connerney et al., 2018) as well as possible atmospheric effects such as a more opaque atmosphere (Ozak et al., 2010). The polar projected 2D histograms contain no information on the varying opacity of the ionosphere and therefore makes the latter difficult to determine from the Chandra data alone. Therefore, this is not the main focus of the study but should be considered in future work.

The overall morphology of the brightest SHS emissions is found to be more spotlike (i.e. constrained in $\sim 350^{\circ}-60^{\circ} \mathrm{S} 3$ lon and $\sim-60^{\circ}$ poleward in latitude) when compared to its northern counterpart. The spreading of the SHS extends just beyond the Ganymede footprint (as shown between an S3 longitude of $\sim 45^{\circ}$ and $180^{\circ}$ ) similar to the NHS. This suggests that the SHS morphology may be very variable across the observations or may be another consequence of poorer viewing conditions. The unfavourable viewing conditions may impact the accuracy of the SHS mapping. 
The average powers, energy flux and maximum brightnesses for the North and South auroral emissions throughout our catalogue are shown as histograms in Figure 2. The values for the mean $(\mu)$, median $(M)$ and standard deviation $(\sigma)$ are displayed in each panel with $\mu$ and $M$ plotted as the solid and dashed vertical lines respectively. The median is calculated for each distribution as the shortest duration observation $(\sim 3$-hr observation, ObsID 18676) produced an unusual maximum auroral brightness in both polar regions (as shown in Figures 2c) and f)). For the power and flux calculation for each observation, we assume a photon energy of $\sim 0.5 \mathrm{keV}$ (halfway between the sulfur and oxygen emission lines), similar to previous work (e.g. Dunn et al. (2016, 2017); Gladstone et al. (2002)). The energy flux we calculate here is the X-ray flux observed from Chandra (i.e. at Earth), accounting for the changing Chandra-Jupiter distance over the 20 year period. We assume that the North and South auroral emission regions account for $\sim 10 \%$ and $\sim 5 \%$ of Jupiter's disk respectively, which is typical of what we observe from the Chandra image data. The counts, duration of observation, average angular diameter of Jupiter and Chandra-Jupiter distance used in our calculations are shown in Tables S1 and S2 in the Supplementary Information. We note that the powers and energy fluxes calculated for the South are a lower limit due to the poorer viewing geometry which decreases the number of counts detected by Chandra.

As shown in Figures 2a) and d), the mean X-ray auroral power throughout the catalogue was found to be $\sim 1.95 \mathrm{GW}$ and $1.44 \mathrm{GW}$ for the North and South respectively within the auroral regions defined in Section 2. All our results using the power and flux calculations are shown in Table S2. The standard deviations, $\sigma$, for all of the distributions representing the southern emissions are found to be smaller than their northern counterparts. This may suggest that the driver producing the southern auroral X-rays and SHS are less variable than those responsible for the northern emissions. The different driver may also contribute to the more diffuse emissions we observe in the South.

The auroral powers were found to correspond to an average flux of $2.92 \times 10^{-13}$ erg $\mathrm{cm}^{-2} \mathrm{~s}^{-1}$ for the North and $2.14 \times 10^{-13} \mathrm{erg} \mathrm{cm}^{-2} \mathrm{~s}^{-1}$ for the South. The mean maximum auroral brightness was observed to be $1.48 \mathrm{R}$ (Rayleighs) and $0.62 \mathrm{R}$ respectively, again reflecting the brightness asymmetry between the poles shown in Figure 1. The observations throughout the catalogue varied in duration depending on the science focus, which may have an effect on the values we calculate here. From the 29 HRC-I observations, 6 were optimized for viewing of the intense hot spot region in the north with a duration of $<1$ jovian rotation. The remaining campaigns lasted for one jovian rotation or more to explore, in detail, the full X-ray emissions. For the rest of this study, we focus in detail on the northern emissions.

\subsection{Exploring the persistence of concentrated NHS auroral photons}

The average maps in Figure 1 hint at the morphology of the northern auroral Xrays, and the structure of the typical northern hot spot embedded in that region, but in this section we apply some quantitative criteria to define where photons are concentrated. We build on the method of (Weigt et al., 2020) and define a so-called hot spot region across the vast majority of the catalogue. This numerical criterion consists of a spatial select region of the hot spot position in the North (S3 longitude: $100^{\circ}-240^{\circ}$, latitude: $40^{\circ}-90^{\circ}$, as stated in Section 2) and a numerical threshold on photon concentration ( $>7$ photons per $5^{\circ} \mathrm{S} 3$ lon $\times 5^{\circ}$ lat) within the NHS. From the Chandra HRCI catalogue, 26 out of the 29 observations had NHS X-ray emissions that were within the criterion threshold. Two of the observations (ObsID 15670, 18676) had insufficient counts to produce the more highly concentrated NHS emissions. Figure 3 shows plots of a 2$\mathrm{D}$ histogram from the resulting emission on a $3^{\circ} \mathrm{S} 3$ lon $\times 3^{\circ}$ lat grid and projecting onto a planetographic polar map. These plots allow us to determine the typical location of the X-rays concentrated within the NHS. The 1-D histograms of S3 longitude and latitude shown in panel (a) provide a clear representation of the width of the average hot 




Figure 3. (a) Cartesian plot (S3 longitude vs. latitude in degrees) with the number of photons represented as a 1-D histogram of S3 longitude and latitude. The corresponding polar planetographic projection of the NHS X-ray emissions is found using the criterion adapted from Weigt et al. (2020) is shown in (b). The polar plot is of similar format to Figure 1 with binning of $3^{\circ} \mathrm{S} 3$ lon $\times 3^{\circ}$ lat. The same binning is used for the histograms, showing more clearly the width of the average hot spot. The Io and Ganymede footprints are plotted in both panels to provide context on the approximate location of the NHS driver. The color bar represents the percentage X-ray photons found across all observations within the spatially select region with the photon concentration threshold applied (26 out of 29 observations from the catalogue). The color bar used in both panels shows what percentage of the observations contained NHS X-ray emissions in each bin. The concentrated X-ray emissions occurring in all observations (100\%) in a selected region are denoted by the cross-hatched area in all panels.

spot and highlights the variability within the region. The color bar represents the percentage of observations that had X-rays mapped to a $3^{\circ} \mathrm{S} 3$ longitude $\times 3^{\circ}$ latitude bin from $0-100 \%$. As highlighted by the cross hatched regions in Figure 3 , the NHS always appears in the range $\sim 162^{\circ}-171^{\circ} \mathrm{S} 3$ longitude and $\sim 60^{\circ}-66^{\circ}$ latitude. This region of interest will be herein referred to as the "averaged hot spot nucleus" or AHSNuc (i.e. with photon concentrations above threshold in $100 \%$ of the observations). As the AHSNuc region is found in all observations, this region may map to the location of a physical driving process that is always turned on within the jovian magnetosphere.

From the catalogue of observations, we find that the hot spot often appears (i.e. occurs $70-99 \%$ ) in the range $\sim 153^{\circ}-183^{\circ} \mathrm{S} 3$ longitude and $\sim 57^{\circ}-72^{\circ}$ latitude, and typically surrounds the AHSNuc. The regions here are found to accompany the central emission throughout the catalogue through possible movement of the hot spot. This would therefore suggest that the driver producing the more intense NHS emissions is often variable, leading to a possible change in morphology and hot spot position. This is further highlighted in the regions where we find that the hot spot is occasionally (i.e occurs between $30 \%$ and $70 \%$ ) found. The emissions here are located at $\sim 54^{\circ}-75^{\circ}$ latitude and span a slightly larger range of longitudes $\left(\sim 150^{\circ}-195^{\circ} \mathrm{S} 3\right.$ longitude $)$, falling away from the AHSNuc. 


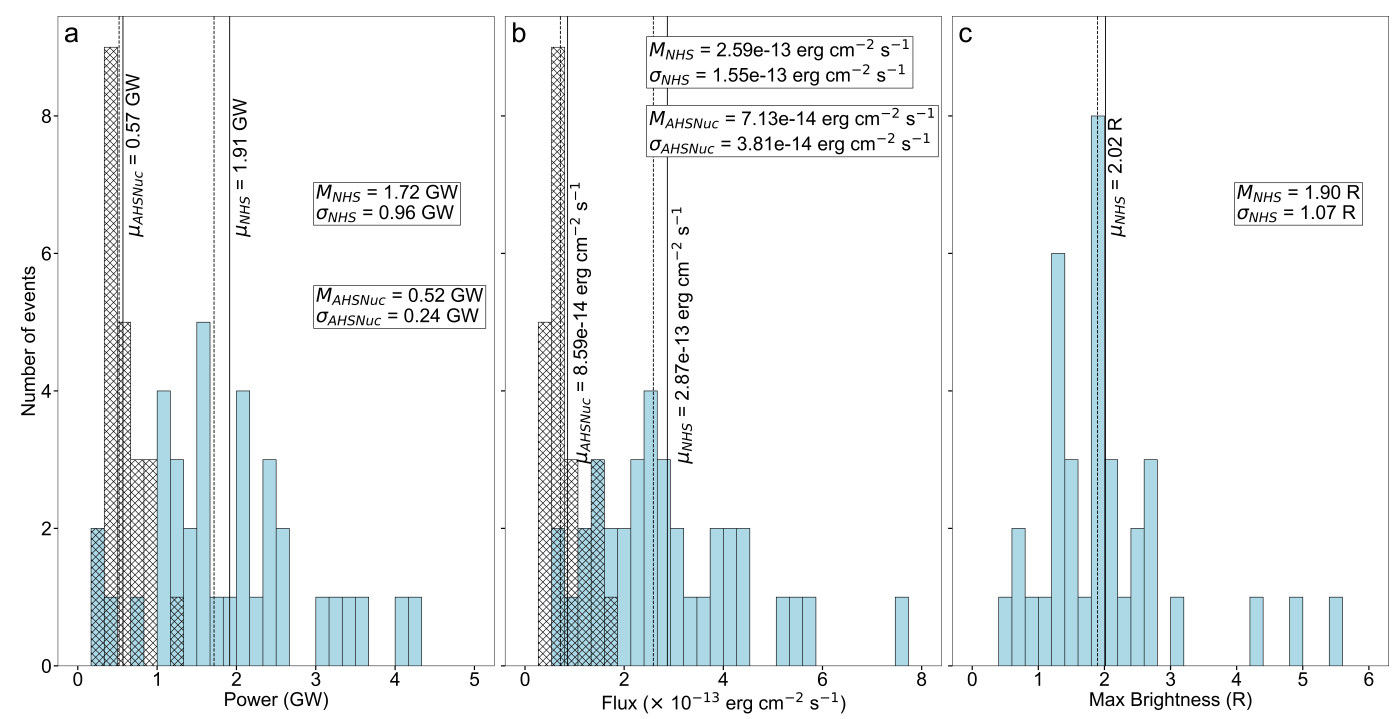

Figure 4. Histograms of the same format as Figure 2, showing the average power, flux and maximum brightness of the NHS (blue) and the AHSNuc (cross-hatched). Any overlap of any parameters in the catalogue are shown by the blue, cross-hatched in the histograms. The median and standard deviation values for both regions are shown on the plot. The mean for both the NHS and AHSNuc are shown by the vertical solid lines with the corresponding value displayed alongside. The median for each region is shown by the dashed vertical line. The maximum brightness of the AHSNuc is not shown in panel (c) as finding an accurate brightness over a very small area is difficult to obtain using our current method. The overall average brightness of the AHSNuc can be interpreted from Figure 1

The remaining hot spot locations ( $<30 \%$ occurrence) are found to be rare using the set criterion and considered extreme hot spot behaviour. From Figure 3 it is clear that these regions are more equatorward (beyond the Io footprint in many regions) and span the entire longitude range of the Cartesian grid $\left(\sim 120^{\circ}-237^{\circ} \mathrm{S} 3\right.$ longitude $)$. These regions may be a result of other magnetospheric process being activated during the time of the observations which may only occur under certain conditions, eluding to a possibly more fragmented hot spot. The decreasing gradient of the color bar in Figure 3 clearly illustrates the variable morphology of the NHS emission across all observations and can allow us to analyze further the typical and extreme behaviour of the X-ray auroral emissions.

We apply the same methods described in Section 3.1 to the NHS and AHSNuc to produce histograms of the auroral power, flux and maximum brightness in these auroral features throughout the catalogue (Figure 4). The histograms are of identical format to Figure 2. For our calculations, we assume that the emissions observed in the concentrated NHS and AHSNuc cover $\sim 7 \%$ and $\sim 1 \%$ of the jovian disk respectively. This was found by comparing the auroral feature in Figure 3 to the overall averaged emissions in Figure 1. From Figure 4 we find that the AHSNuc contributes to $\sim$ one quarter of the entire auroral power of the concentrated NHS region (0.56 (AHSNuc) : 1.91 (NHS) GW) and $\sim$ one third of the auroral flux $\left(0.86: 2.87 \times 10^{-13} \mathrm{erg} \mathrm{cm}^{-2} \mathrm{~s}^{-1}\right)$. These powers and fluxes of the NHS correspond to a mean maximum brightness of 2.02 R, representing the brightest part of the jovian X-ray spectrum. The standard deviation of the AHSNuc auroral power and flux distribution is less than that found for the NHS, suggest- 
ing the driver producing the AHSNuc is less variable. The difference of the distributions for all auroral properties across the NHS and ANHSNuc suggest that multiple drivers producing the X-ray auroral emissions may be plausible. These results suggest that the AHSNuc may be a key auroral feature within the NHS which behaves differently from the full auroral region and must be taken into account in future X-ray auroral studies. However, we do note these results will be further improved with future scheduled Chandra observations and provide more accurate statistics.

The average brightness of the AHSNuc can be inferred from the averaged hot spot emission in Figure 1a). The maximum brightness of the AHSNuc is not shown in Figure 4 as finding an accurate brightness over a very small area is difficult to obtain using our current method. We do note that the three intervals $2-3 \sigma$ greater than the mean NHS brightness (ObsID 18678 NHS1, 15671 NHS1 and 18301 NHS1) all correspond the shortest exposure times of the NHS. Similar to the extreme case identified in Figure 2, the shorter exposures times produce more unusual values for the auroral brightness. All results from our calculations of the NHS and AHSNuc for the entire catalogue are shown in Tables S3a and S3b.

The most extreme case of variable morphology was found by Chandra during a 20-hr ( 2 Jupiter rotations) observation on 28 February 2017 (ObsID 20000) during Juno's fourth apojove (AJ4). With the longer exposure time, Chandra-HRCI is able collect more photon data. From the criterion, the concentrated emissions were observed over a vast range of longitudes (S3 lon: $\sim 120^{\circ}-237^{\circ}$ ) and latitudes (lat: $\sim 39^{\circ}-75^{\circ}$ ). The X-ray aurora within the NHS emitted a power of $\sim 3.24 \mathrm{GW}$ (Table S3a). Comparing these numbers with the only other observation that had a duration of $\sim 2$ Jupiter rotations (ObsID 2519, 25 February 2003), the X-ray aurora within the NHS is found to be $\sim 7$ times more powerful $(0.465 \mathrm{GW})$ and the region $\sim 4$ times larger in longitude. As both observations occurred at roughly the same time of year, the seasonal changes between both intervals are very small. Therefore this suggests that the changes in morphology and X-ray power are most likely caused by a change in magnetospheric conditions due to the solar wind or internally from Io.

Another notable observation showcasing the extreme behaviour of the hot spot was found during a 7-hr observation (ObsID 22159, 8 September 2019) in tandem with Juno perijove (PJ22), optimized for NHS viewing. The emissions here were found to lie within the kink of the GAM Ganymede footprint and extended beyond the Io footprint and were $\sim 2 \sigma$ more powerful $(4.03 \mathrm{GW})$ than the calculated mean power. The only interval that had more powerful auroral emissions was during the second NHS interval during a 11hr observation (ObsID 18608, 24 May 2016) at 4.24 GW. The hot spot emission observed from ObsID 22159 was found to reside in a small region (S3 lon: $\sim 135^{\circ}-180^{\circ}$; lat: $\sim$ $48^{\circ}-66^{\circ}$ ) with the AHSNuc lying on the edge of the emission. This shows that the driver producing the emissions can also cause variation in the position as well as morphology. The hot spot from ObsID 18608 was found to be located in a similar position to ObsID 22159 with a slightly elongated morphology. The plots of each of the extreme cases mentioned here and all other observations are shown in the Supplementary Information provided (Figure S3). The plots are of the same format as Figure 3 with the color representing the number of photons found in each bin.

\subsection{Mapping hot spot photons to their magnetospheric origins}

In order to map the origin of highly concentrated X-ray emissions of the NHS shown in Figure 3, we use the Vogt et al. $(2011,2015)$ flux equivalence mapping model. The model relates a region in the ionosphere to source region in the equator. This method assumes that the flux through a given region is located in the jovigraphic equator, which is calculated from the Galileo catalogue with a 2-D fit (radial distance and local time (LT)). The equatorial flux in a given region found from the fit to the data should therefore be 
equivalent to the flux through the region in the ionosphere to which it maps. The mapping model has a strong dependence on subsolar longitude (SSL) of the photons. The mapping model inputs are the ionospheric position (in S3 lon and latitude) and the SSL of the time-tagged X-ray photons, which we obtain from the mapping algorithm discussed in Weigt et al. (2020). In this study, we use the Vogt et al. model with the internal field from GAM. This field model was selected as GAM fits the Ganymede footprint best in the north better than VIP4 or VIPAL (Hess et al., 2011) (excepting JRM09 (Connerney et al., 2018)). This kink arises from a localized quadrupolar term, introduced in the magnetic field to reproduce the anomaly at the North pole. This will have an effect on the more intense regions of the NHS, where the emissions map to in the magnetosphere and how we interpret our results.

Figure 5a) shows a statistical map of the origins of all mappable photons from Figure 3, using the GAM field model and the SSL for each individual photon. A statistical map of the location of the possible driver producing the AHSnuc is shown in Figure 5b). Their corresponding exposure maps are shown in Figures 5c) and (d), where the number of counts are normalized by the length of the observation window. All plots consist of a 2-D histogram, showing the number of mapped events ((a) and (b)) and average number flux ((c) and (d)), represented by the color bar. The Joy et al. (2002) model limits for both a compressed (solar wind dynamic pressure of $0.306 \mathrm{nPa}$ with subsolar distance $\sim 60 \mathrm{R}_{J}$; black-dashed line) and expanded (0.039 $\mathrm{nPa}, \sim 90 \mathrm{R}_{J}$; solid black line) magnetosphere are also plotted to provide context to the mapped origins of the X-ray emissions. This model combines the observations from multiple spacecraft (Pioneer 10 and 11, Voyager 1 and 2, Ulysses and Galileo) which crossed Jupiter's magnetopause boundary with a magnetohydrodynamics simulation to infer the dynamic pressure of the upstream solar wind and associated subsolar standoff distance. The mapped events have been binned by a radial distance of $10 \mathrm{R}_{J}$ and 1 hour LT. From Figure 5a), it is clear that two main populations arise from the analysis: one concentrated on the noon sector, and a larger population spread across pre-dusk to pre-midnight of the magnetosphere (15 LT - $21 \mathrm{LT}$ ), even when corrected for exposure time (Figure 5c)). The majority of events in both populations lie close to, in between, or on the magnetopause boundary (either expanded or compressed). The population that lies on the pre-dusk to pre-midnight magnetopause boundary consists of $\sim 40 \%$ of all mapped photons in the catalogue, suggesting that this sector of the magnetosphere is the optimum location for the driver of ions needed for SXR production. The wedge of high photon counts at 18 LT across all radial distances, shown in Figure 5a), disappears in the corresponding exposure map. This region was found to be mainly dominated by one observation, ObsID 20000, where the most extreme hot spot behaviour was found, as discussed in Section 3.2.

The driver producing the AHSnuc is found to lie between noon and 20 LT (Figures $5 \mathrm{~b}$ ) and (d)) and consists of $\sim 7 \%$ of all mapped photons in the catalogue. This population is also found to lie between both magnetopause boundaries, therefore suggesting that the X-ray driver for the NHS may be sensitive to possible fluctuations in the magnetopause location.

The Vogt flux equivalence model is built from Galileo data, where the model algorithm is valid from $\sim 15 \mathrm{R}_{J}$ (Ganymede footprint) to $\sim 150 \mathrm{R}_{J}$ (beyond which there are insufficient data) and is sensitive to possible changes in ionospheric position. Using the flux equivalence model and the same internal field as shown in Figure 5, we estimate the errors in mapping that are propagated through from the uncertainty in X-ray photon placement. We apply the same $2.5^{\circ}$ shifts in latitude and S3 longitude to a grid of simulated photons with the same sub-solar longitude (SSL). The resulting plots are shown in Figure S1 in the Supplementary Information, illustrating the positions of the original and shifted mapped photons from the grid (in both latitude and longitude separately). The shifts used in this study are more extreme than we may observe using the Chandra HRC-I instrument. The diameter of the Gaussian PSF of the instrument is smaller 

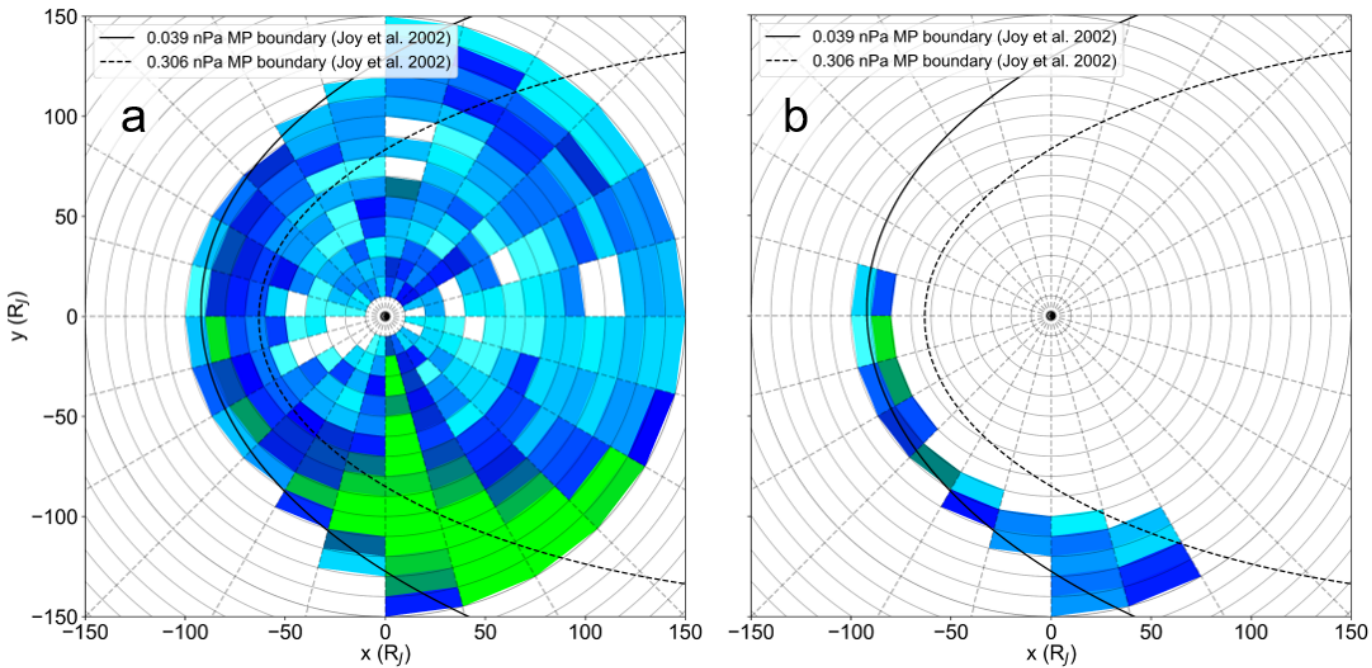

$\begin{array}{llccccccc}0 & 5 & 10 & 15 & 20 & 25 & 30 & 35 & 40\end{array}$
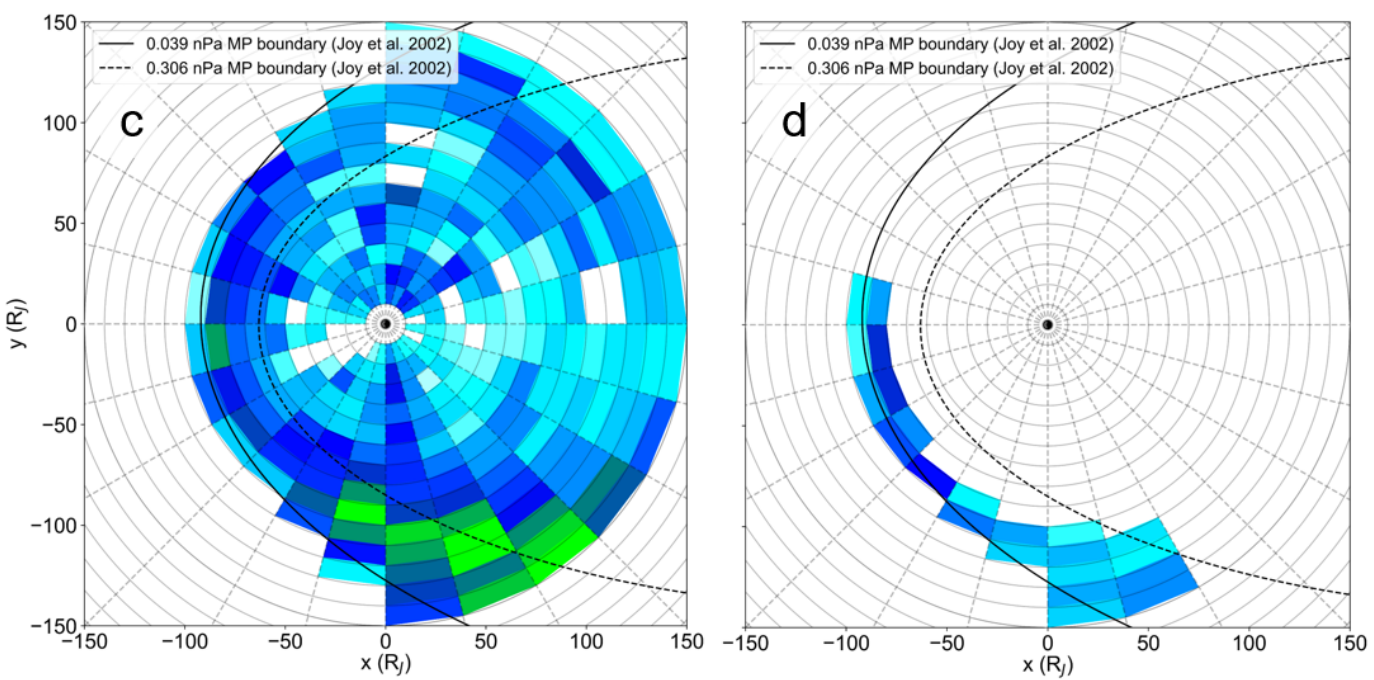

$\begin{array}{llllll}0.00 & 0.25 & 0.50 \begin{array}{r}0.75 \\ \text { counts/s }\end{array} & 1.00 & 1.25 & 1.50 \\ \times 1.75 \\ \times 10^{-3}\end{array}$

Figure 5. Plot of (a) all the mapped photons and (b) the mapped origin of the AHSNuc photons within the threshold for 26 out of 29 observations. The corresponding exposure maps are shown in (c) and (d), where each photon mapped has been normalized by the length of window observed for each event in the catalogue. The photons are mapped using the Vogt flux equivalence mapping model using GAM. The concentric circles in (a) - (d) represent the distance from Jupiter in $10 \mathrm{R}_{J}$ increments. The Joy et al. (2002) compressed (black dashed line) and expanded (solid black line) magnetopause boundary limits are also plotted. The mapped data are binned by a radial distance of $10 \mathrm{R}_{J}$ and a local time (LT) of 1 hour. The color bar represents the number of events found (panels (a) and (b)) and the average number flux (counts/s) in each bin (panels (c) and (d)). 
than the $2.5^{\circ}$ shift used here as we can resolve the center of the PSF (photon ionospheric positions) to $1^{\circ} \mathrm{S} 3$ lon $\times 1^{\circ}$ lat. From comparing both panels, a shift in either latitude and S3 longitude results in different changes in both radial distance and local time depending on where the origin is within the jovian magnetosphere. This means that mapped photons that lie on or close to a magnetopause boundary may be interpreted as beyond or within the magnetopause region; a caveat we take into account when interpreting our results. The magnetopause is also not a static location and so mapping to it is not exact (using any model). The mapping uncertainty from ionospheric position will therefore be affected by magnetospheric conditions as well as the strong dependence on SSL. Therefore calculating the full error on mapping is very difficult and not the main focus of this study.

As the flux equivalence model uses Galileo data, where the magnetosphere was mainly expanded or returning to an equilibrium state throughout the campaign, observations during a compression are more difficult to model. As a result, we interpret events in between both Joy model limits and close to the compressed boundary to lie in a region on the magnetopause boundary or just outside the magnetosphere. It is therefore clear that, on average, most of the intense NHS emission is found to originate on/near the magnetopause boundary pre-dusk to pre-midnight. Vogt et al. (2019) highlight that a compression event can contribute to a shift in ionospheric position of the main auroral emissions towards the jovian magnetic pole. Such shifts can change the magnetospheric mapping of the static (non time dependent) flux equivalence model of up to tens of $\mathrm{R}_{J}$. This effect, in addition to the strong SSL dependence, may be responsible for the spread of the main mapped drivers in Figure 5 on the noon and dusk boundary. Finally, we show comparisons with an applied shift in ionospheric position and compare to JRM09 in the Supplementary Information provided (Figure S2). This shows how the interpretation of the driver may be affected depending on the field model used in concert with the Vogt et al. flux equivalence model.

\subsection{Searching for quasi-periodic NHS emissions}

Following the Rayleigh test techniques outlined in Jackman et al. (2018) and Weigt et al. (2020), we search for quasi-periodicity or quasi-periodic oscillations (QPOs) in the catalogue. Figure 6 show the results of the timing analysis for the QPOs found within the (a) NHS region and (b) AHSNuc. The QPOs identified with a significance below our $99 \%$ significance threshold or $p$-value $(p)>0.01$ are represented by the gray distribution. The $p$-value here is defined to be the probability of obtaining results at least as extreme as the observed data assuming a correct null hypothesis, in this case no periodic signal. Any QPOs found from the timing analysis with statistical significance $\geq 99 \%$ ( $p$ $\leq 0.01)$ are shown in solid blue and green for the NHS and AHSNuc regions respectively. The striped distributions represent QPOs found with significance $\geq 99.999 \%\left(p \leq 10^{-5}\right)$. The results for all observations used in the Figures $3-6$ for the NHS and AHSNuc are show in Tables S4 and S5 in the Supplementary Information.

The Rayleigh test was carried out for each interval the concentrated X-ray emissions were detected by the instrument during the observation (Tables S4 and S5). This therefore allows us to determine each time the NHS is in view by setting a limit of the time interval between the time-tagged photons. We set a time limit of $>180 \mathrm{~min}$ between time-tagged photons to define each time the NHS is in Chandra's field of view. The duration of each viewing of the NHS, average Chandra-Jupiter distance over the interval, total counts and count rate are given to allow us to ensure there were enough photons detected to produce a power spectrum that represented the Chandra data well. Any observations with counts $<30$ were removed from the analysis. The next columns show the proportion of photons mapped in these regions, shown in Figure 5. From both tables, $\sim 90 \%$ of observations have $<50 \%$ of photons mapped using the Vogt et al. (2011, 2015) flux equivalence model. This may be due to the fact that much of the NHS and 




Figure 6. Histogram of the Rayleigh test results from the (a) full NHS and (b) AHSNuc throughout the catalogue (as shown in Tables S4 and S5). The histogram is of identical format to Figures 2 and 4. The gray distribution on both panels represents the QPOs found throughout the catalogue with a statistical significance $<99 \%$ or $p$-value $(p)>0.01$. The solid blue and green bars show the number of QPOs with significance $\geq 99 \%(p \leq 0.01)$ and the striped bars represent a $p \leq 10^{-5}$ (significance $\geq 99.999 \%$ ).

AHSNuc either maps outside the model constraints $\left(<15 \mathrm{R}_{J}\right.$ or $\left.>150 \mathrm{R}_{J}\right)$ and/or the mapping is limited by the SSL during the observation, resulting in poorer viewing conditions for most observations.

The timescales of the significant QPOs throughout the catalogue are found to be $\sim 3.9-36.4 \mathrm{~min}$ and $\sim 2.3-22.4 \mathrm{~min}$ for the NHS region and AHSNuc respectively. The difference in QPO ranges are a result of down-selecting from the larger, full NHS region to the smaller AHSNuc, a specific feature in the auroral emissions. The change of periods show that in many cases the full hot spot auroral region does not pulsate simultaneously and that smaller structures within the hot spot can pulsate independently form the surrounding auroral emissions. As shown in Tables S4 and S5, the longest QPO, with $p \leq 10^{-5}$, was found during the first interval the NHS was observed on 18 June 2017 (ObsID 20001) and is in agreement with the results found by Weigt et al. (2020). The only QPOs found from the AHSNuc $>2 \sigma$ of the mean period were from ObsID 22146 ( $\mathrm{sim}$ 7-hr observation optimized for hot spot viewing on 13 July 2019) and ObsID 20733 NHS2 (second NHS interval of $\sim 11$-hr observation on 1 April 2018) at 21.7 and 22.4 mins respectively. We find in many observations, the NHS and AHSNuc are found to both produce significant QPOs during the same interval (e.g. ObsID 15669, 18677, 22146). During three intervals (ObsID 16299 NHS2, 20002, 20733 NHS2) the NHS and AHSNuc were found to produce the same significant QPO, suggesting that the dominant driver(s) producing the auroral emissions were associated with the AHSNuc.

Many of the QPOs found here agree with the values found by the timing analysis study of Jackman et al. (2018). In their study they noted that differences in QPO period (and associated significance) are highly sensitive to the selection of the hot spot. Their work explored the entire northern (and southern) auroral region, with a simple downselect for hot spot based on viewing a time window as the hot spot traversed the disk. Here we employ a more strict spatial criterion for hot spot selection, and, while for most 
examples, our results are broadly in line with those of Jackman et al. (2018), there are examples where the period and the significance differ. This shows how sensitive the QPOs are to the selection of the hot spot - and thus in turn, perhaps, how tightly constrained the driver of the periodic emission is. We also note that there is no clear correlation between the average Chandra-Jupiter distance and detection of significant QPOs in both the full auroral region and the AHSNuc (Tables S4 and S5) as well as any distance dependent auroral parameters (i.e. flux, power). We would expect the closer Jupiter is to the instrument, the easier it would be to detect significant QPOs with brighter and more powerful aurora which we do not observe here. Therefore, we can rule out distance as a parameter than can influence detecting the X-ray emissions and inhibit our timing analysis to detect statistically significant QPOs.

We further improve the significance of the signals found here by testing the sensitivity of each of the light curves to the observed frequency of the signal. We do this by using a Jackknife test (Quenouille, 1949, 1956), by removing a number of photons from each of the light curves and running the Rayleigh test algorithm, using an identical frequency space, on each new light curve (Efron \& Stein, 1981). All the power spectra generated are then plotted together and the time interval between the minimum and maximum period found, $\Delta P$, is measured. This allows us to provide an estimate of the sensitivity of each light curve to frequency. As Chandra has a poor throughput and therefore observes very few photons, the Jackknife test used in this study removed a maximum of two photons each time, ensuring that there was no degeneracy from the selection process. Tables 1 and 2 show the results of the Jackknife test for the removal of one photon (JK1) and two photons (JK2) for each of the QPO datasets shown in Figure 6 above our $99 \%$ significance threshold. The first column in both tables gives the unique Chandra ObsID for each observation. The following columns gives the region and interval during the observation window (i.e. NHS2 $=$ NHS observed for the 2nd time, and similarly for AHSNuc) and the results from JK1 and JK2. The nomenclature and formatting are similar to Tables S3 and S4 in the Supplementary Information. All the hot spot observations with a $\Delta P>5 \mathrm{~min}$ are bold text. These QPOs, although statistically significant from the Rayleigh test, are found to be not robust and highly sensitive to frequency. As a result, we remove these periods from the catalogue, reducing the significant QPOs from 14 to 12 for the NHS region and 17 to 9 for the AHSNuc. The light curves found for the AHSNuc contained far fewer photons and are therefore more sensitive to the Jackknife test. However, we do note that this test does not account for the coherence (i.e. how sinusoidal) of the QPO signal. The more coherent signals will produce a smaller $\Delta P$ value from both Jackkinfe tests. Therefore some of the QPOs removed from the catalogue may still be robust but with a non-sinusoid envelope. Future temporal studies may want to consider the coherence in their timing analysis to avoid the possible bias from such tests, although this is non-trivial to implement when used with the Rayleigh test.

The range of quasi-periods found from our catalogue may correspond to a variety of possible drivers. The vast range in significant QPOs found suggest that the X-ray driver may be connected with ultra-low frequency (ULF) waves along the magnetopause boundary. Pulsations $\sim 5-60$ min from standing Alfvén waves have been found throughout the jovian magnetosphere (Manners et al., 2018). The QPOs produced by the AHSNuc may be associated with possible pulsed dayside reconnection on the magnetopause. Bunce et al. (2004) found that such reconnection could produce pulsations of $\sim 30-50$-min and is more active during magnetospheric compressions. This therefore may be responsible for the larger QPOs found in our catalogue. Combining both our timing and mapping results, we suggest that there are multiple drivers producing the X-rays along the magnetopause boundary from noon to the dusk flank. Figures 5 and 6 show the possibility of strong contributions from multiple drivers which may either be semi-permanent or more sporadic in nature. 





نे 


\section{Discussion}

The results of our statistical study analyzing the Chandra HRC-I dataset allows us, for the first time, to explore in detail the statistical significance of the variability in morphology of the X-ray emissions and their origin. We adapt the Weigt et al. (2020) numerical criterion to define the highly concentrated NHS emissions, allowing us to hone on the QPO regions and their associated magnetospheric drivers.

\subsection{Characteristics and polar conjugacy of auroral X-ray emissions}

The polar plots and histograms we present in Section 3.1 clearly show an asymmetry in brightness and morphology across the catalogue between the North and more diffuse South. This asymmetry has been observed in previous case studies (e.g. Dunn et al. (2017); Weigt et al. (2020)) and is believed to possibly result from a combination of unfavourable viewing geometry of the south due to Jupiter's tilt (Dunn et al., 2017); the radically different magnetic field strength and topology at the poles found by Juno magnetometer data (Connerney et al., 2017, 2018) and the opacity of the jovian atmosphere (Ozak et al., 2010). The North polar region is observed to have a non-dipolar field topology and is more than twice as strong as the more dipole-like South pole (Moore et al., 2018). The difference in magnetic field magnitude may effect the mechanism(s) that allow the ions to be injected into the ionosphere at the poles. The most plausible explanation for this arises from the stronger non-dipolar north producing a stronger mirror force than its southern dipolar counterpart. This will produce the large potential drop required to accelerate the ions (both solar wind and iogenic in origin) to the larger energies needed for ion precipitation in the ionosphere to produce the X-ray aurora (Cravens et al., 2003; Houston et al., 2020). This process may favour the slightly extended teardrop morphology we observe here in the brightest North emissions. Since the configuration of the North polar region is more non-dipolar and producing a stronger magnetic field strength, the mirror force would be greater. This would lead to more ions being trapped, leading to more ions being accelerated to the energies required for precipitation than in the South. A similar mechanism may operate in the South where the mirror force will be weaker and therefore fewer ions will be accelerated to the required energies for precipitation, leading to dimmer X-ray emissions.

Recent work by Dunn et al. (2020a) classified the X-ray aurora into three categories from Chandra and XMM-Newton observations in 2007: hard X-ray (energies > $2 \mathrm{keV}$ ) bremsstrahlung main emission; pulsed SXR emissions (both regular and irregular) and dim flickering (quasi-continuously present emission, varying on very short timescales). They identified that the X-ray emissions were dominated by pulsed SXR emissions, mainly produced from iogenic ions. They found that the brightest X-ray aurora coincided with magnetospheric expansions and was found to have a more patchy and extended morphology. The aurora during a compression was found be more concentrated into a localized bright region at S3 longitudes of $\sim 160^{\circ}$ to $180^{\circ}$. The polar plots of the extended North emission reflect this behaviour across the catalogue, showing the variation of the magnetospheric conditions throughout the catalogue. The extended emission is found to be more spread and diffuse with a localized bright tear-drop around $180^{\circ} \mathrm{S} 3$ lon in the center (see Figure 1). The brightest emission residing within this tear-drop region lies in roughly the same location as the core region of the X-ray emission, observed by Kimura et al. (2016) during an UV and X-ray campaign in 2014. Therefore this region may be a recurring characteristic of the X-ray auroral emissions.

Many previous case studies have analyzed the X-ray emissions during times of compression (Dunn et al., 2016; Wibisono et al., 2020; Weigt et al., 2020). They found localized brightenings within the northern auroral emissions (Dunn et al., 2016) and an extended morphology (Weigt et al., 2020) during a compression event. Wibisono et al. (2020) found that iogenic ions are responsible for the emissions with very little contri- 
bution from the solar wind during magnetospheric compression. Kimura et al. (2016) however found that the count rate of the core region during the 2014 campaign was positively correlated with the solar wind velocity as opposed to morphology. The flux within this region however may change due to the changing dynamic pressure caused by the solar wind's effect on the magnetosphere as opposed to a direct effect on the X-ray emission itself. Therefore, the variable morphologies we see in the northern X-ray aurora (as classified by (Dunn et al., 2020a)) may be a result of changing dynamic pressure and reflect the jovian magnetosphere's sensitivity to such changes.

\subsection{Morphological variability and origins of the concentrated NHS emis- sions}

The polar projected 2-D histograms of hot spot location and histograms of the auroral properties in Section 3.2 depict the typical and extreme behaviour of the concentrated NHS X-ray emissions. For the first time, we find a statistically significant region in the NHS emission, AHSNuc, using the numerical threshold previously defined. The less variable AHSNuc (Figure 4) provides further evidence supporting the X-ray emissions are highly concentrated, which can be mapped to specific driver mechanisms. External mechanisms, like the solar wind ram pressure, may affect the morphology of the emission surrounding the AHSNuc in the same way as the averaged X-ray auroral emissions.

The typical behaviour of the NHS (occurrences of $>70 \%$ in the catalogue shown in Figure 3) is found to be confined within an ellipse of semi-major axis $\sim 15^{\circ}$ and semiminor axis $\sim 7.5^{\circ}$, centered at $\left(168^{\circ} \mathrm{S} 3 \mathrm{lon}, 65^{\circ}\right.$ lat $)$. Within this region, the gradient of the photon occurrence is found to vary at higher longitudes away from the AHSNuc. This may be evidence of further segregation that has been alluded to occur during a compression event (Dunn et al., 2016; Weigt et al., 2020) and may be the locations for concentrated X-ray emission to brighten during these times, as found by Dunn et al. (2020a). The more extreme behaviour (occurrences $\leq 20 \%$ ) we observe from the NHS emissions surrounds the ellipse defining more typical behaviour. This region of extreme behaviour may be a result of a lower solar wind dynamic pressure causing an expansion of the magnetosphere. Therefore our study suggests very few X-ray observations in the catalogue coincided with an expansion event.

Figure 5 shows the resultant mapping using the Vogt et al. (2011, 2015) flux equivalence model with the Grodent Anomaly Model (GAM) (Grodent et al., 2008) option. The model finds two ion populations along the magnetopause boundary when mapping the NHS: a significantly large population in the pre-dusk to pre-midnight sector, coincident with the dusk flank and a smaller cluster at noon. The former population identified in this study agrees with previous work using the Vogt model to determine the origin of the NHS (Kimura et al., 2016; Dunn et al., 2017; Weigt et al., 2020). The driver producing such emissions was suggested to be related with Kelvin-Helmholtz instabilities (KHIs) on the dusk flank. KHIs along the magnetopause boundary are responsible for energy, momentum and plasma transfer between the magnetosheath and the magnetosphere. Such phenomena have previously been observed at Jupiter's magnetopause boundary (Delamere \& Bagenal, 2010; Desroche et al., 2012) where the velocity shear between solar wind flow and sheath flow is greatest. These instabilities are predicted to be predominantly found on the dusk side of the boundary at Jupiter (Zhang et al., 2018). This contradicts the expectation where shear flows are expected to be maximized in the pre-noon sector where plasma from the magnetosheath and magnetosphere flow in opposite directions. This has also been observed At Saturn (e.g. (Masters et al., 2012; Delamere et al., 2013)) where it is theorised that the dawn-dusk asymmetry may arise from fast-growing KHIs at dawn being difficult to identify from the spacecraft data in comparison to the more easily detected slow-growing KHIs at dusk (Ma et al., 2015). This 
is consistent with what we observe here as the Vogt et al. flux equivalence model uses Galileo data to trace the origins of the ions in the magnetosphere.

The equatorial conjugate positions in the magnetosphere of both populations identified in this study are also consistent with the location of ultra-low frequency (ULF) activity found by Manners and Masters (2020). The most active regions were found to be near noon at a distance of $\sim 40-100 \mathrm{R}_{J}$ and the dusk-midnight sector, primarily confined along the magnetopause at a distance of $\sim 20-120 \mathrm{R}_{J}$. The power of the ULF waves produced was found to decrease with increasing distance out into the outer magnetosphere, where the X-ray ions are believed to be located ( $>60 \mathrm{R}_{J}$ (Dunn et al., 2016)). KHIs on the magnetopause boundary have been observed to trigger ULF wave activity in Earth's magnetosphere (Hasegawa et al., 2004) and possibly trigger reconnection within the vortices (Nykyri \& Otto, 2001). With the coincident location of the ULF waves and X-ray producing ions, the drivers of the X-ray emissions may be linked to possible ULF wave activity in the jovian magnetosphere.

\subsection{Timescales of possible noon and dusk flank X-ray drivers}

Throughout the literature about the jovian magnetosphere, there have been many theories hypothesizing the driver of the emissions we believe to originate on the magnetopause boundary. In the noon sector, Bunce et al. (2004) proposed a cusp reconnection model as a strong candidate for the X-ray driver, producing $~ 30$ - to 50-min QPOs. The fast flow model predicts that X-ray emissions produced by cusp reconnection will have a brightness, on average, of $\sim$ few Rayleighs $(\mathrm{R})$, which we do observe in the AHSNuc (see Figure 1), up to a few kR (kilo-Rayleighs). We also observe comparable auroral power to the predicted power from the Bunce et al. model. The cusp model may therefore provide a case for the driver we observe on the noon magnetopause boundary. The intensity of the X-ray emissions may be greater than our results suggests due to the poor throughput of the instrument and/or the opacity of the atmosphere (Ozak et al., 2010). Therefore, the AHSNuc may be driven by cusp reconnection and the variable QPOs dependent on reconnection activity, linked to solar wind flow.

Guo et al. (2018) found signatures of rotationally driven magnetic reconnection from magnetometer and charged particle data in Saturn's dayside magnetodisk. They reported multiple reconnection sites and a secondary magnetic island, eluding to a non-steady state process. Such a mechanism may operate in Jupiter's rapidly rotating magnetosphere and produce similar pulsations to those predicted by the cusp model. Magnetic reconnection has been observed on the dawn flank of the jovian magnetopause by Juno (Ebert et al., 2017), where it is believed to play a more significant role in jovian magentospheric dynamics during times of compression (Huddleston et al., 1997). This suggests that both cusp and rotationally driven reconnection may be plausible. Therefore, both reconnection phenomena may be the driver for the noon ion population dominated by the AHSNuc, where the majority of mapped events are found.

Previous studies analyzing the X-ray aurora suggest that the quasi-periodic emissions may be a result of global ULF waves in the magnetic field. ULF waves have been observed ubiquitously throughout the jovian magnetosphere (e.g. (Khurana \& Kivelson, 1989; Wilson \& Dougherty, 2000)) lying within the 10- to 60-min QPO range proposed by Manners et al. (2018) for standing Alfvén waves, and just one possible driver of many suggested possibilities. This ULF period range is similar to what was found in a recent study using a more complicated model to simulate field resonances within the jovian magnetosphere to improve our understanding of Jupiter's magnetospheric response to such magentic fluctuations (Lysak \& Song, 2020).

This type of wave may be a by-product of KHIs on the magnetopause boundary. Both the dayside reconnection processes described by Bunce et al. (2004) and Guo et al. (2018) may be linked to linear sinusoidal KHI waves known as surface waves. These 
surface waves have been observed to drive standing Alfvén waves in the terrestrial ionosphere (Mann et al., 2002; Rae et al., 2005) and could propagate ULF wave activity from the outer jovian magnetosphere to the ionosphere as found by Manners and Masters (2020). Both simulations and observation data suggest that the linear KHI waves on the dayside boundary ( $>10 \mathrm{LT}$ ) may be advected to the dusk flank, in the direction of increasing velocity shear (Zhang et al., 2018; Manners \& Masters, 2020). With the increase in velocity shear, the KHI waves transition from a steady sinusoidal linear wave to a nonlinear KHI wave, with rolled vortices and a greater amplitude. These waves tend to be found in KH-unstable regions on the dawn and dusk sectors of the magnetopause, first suggested by Dungey (1955), where the instability can grow. For the terrestrial case, the thickness and location of such unstable regions are dependent of the angle of the interplanetary magnetic field (IMF) (Farrugia et al., 1998; Foullon et al., 2008). The IMF angle also produces a dawn-dusk asymmetry when the northward field is tilted westwards which may explain the asymmetries we expect at Jupiter (Zhang et al., 2018). At the time of writing, very little has been observed regarding possible KH-unstable regions at Jupiter. Masters (2018) suggested that viscous-like effects, such as KHIs within KH-unstable regions, are likely to dominate over reconnection-type effects at Jupiter compared to Earth. This is in agreement with the possible correlation between X-rays and ULF wave activity we find in this study. With the extension of the Juno science mission, Juno will be located within the dawn-midnight magnetosphere where activity within the dusk flanks can be explored in more detail.

From their extensive study of heritage jovian magnetometer data, Manners and Masters (2020) found ULF QPOs, associated with standing Alfvén waves, spanning 5-60 min across all local times from the Galileo mission (Russell, 1992) and fly-bys performed by Voyager 1 and 2 (Kohlhase \& Penzo, 1977), Pioneer 10 and 11 (Northrop et al., 1974; Sandel et al., 1975) and Ulysses (Wenzel et al., 1992). Galileo observed the jovian magnetosphere across a large span of local times with most of its coverage in the dusk-dawn sector. The QPOs found from the heritage magnetometer data are consistent with the significant quasi-periods we report here. In the kronian magnetosphere, previous studies have found pulsations of $\sim 35-50$ min from possible KHI waves on the dawn and dusk flank of the magnetopause from Cassini magnetometer data (Cutler et al., 2011; Masters et al., 2009). As this lies within the ULF periodicity range, the idea behind lowamplitude ULF wave energy accumulating in the dusk flank from advected waves from the noon sector may be plausible. The mechanism by which ULF wave energy modulates the local ion populations so that they are so energized and pitch-angle scattered into the loss cone is still speculative. The KHIs along the dusk flank may also be reflected by the different X-ray auroral morphologies identified by Dunn et al. (2020a). During compression events, the magnetopause standoff distance is closer to the planet and therefore the dusk flank shrinks. As the boundary is smaller, fewer but more powerful KHI waves may be produced driving the ULF wave activity to produce localized X-ray brightening. The more patchy morphology observed during an expanded magnetosphere may be a result of more vortices generating less powerful KHI waves. This suggests that the "hot spot" may be a result of multiple processes and not confined to a single spot region, as previously theorized. Therefore using such nomenclature, like "hot spot", maybe unsuitable to describe these phenomena.

Our mapping and timing analysis shown here allow for the possibility that multiple drivers, including, but not limited to, cusp/dayside reconnection and KHIs along the noon-dusk magnetopause boundary may be driving the X-ray emission. These drivers may be connected to ULF wave activity which is present throughout the jovian magnetosphere and pulsations similar to those found in our catalogue. The drivers on the noon and dusk magnetopause boundary may be linked to possible ULF wave activity highlighted by Manners and Masters (2020). How they are linked (i.e possible ULF waves from dayside reconnection, advected to the nightside? greater velocity shears on the dusk flank?) is still not fully understood but we have provided the foundations to allow fur- 
ther study into this relatively unknown region. Future studies should consider combining models of the X-ray emissions within the northern auroral region and new in situ observations with Juno's evolving trajectory, moving past midnight toward the dusk flank. This will allow us to delve further into exploring the ULF wave activity on the dusk flank and if it is connected to the pulsating X-ray emissions we observe.

\section{Summary}

From the ever expanding catalogue of Chandra HRC-I observations of jovian Xrays across multiple solar cycles and various solar wind and magnetospheric conditions, we present the first statistical study of its kind to analyze typical and extreme "hot spot" behaviour. We perform mapping and timing analysis techniques to try and determine any statistical significance within the location and pulsations of the hot spot and where they map to in the jovian system. This statistical study included all Chandra HRC-I data to date. We identify a statistically significant region of concentrated X-ray auroral emissions within the hot spot that appear in all observations in the catalogue, the AHSNuc, using the numerical criterion adapted from Weigt et al. (2020). This region maps mainly to the noon magnetopause boundary. All the concentrated X-ray photons that lie within the Weigt et al. (2020) numerical threshold are found to populate the noon magnetopause boundary (dominated by the AHSNuc) and the dusk flank boundary. The results presented here suggest that the X-rays originate from multiple drivers that may be linked to possible ULF wave activity on the magnetopause boundary. The mechanisms we suggest capable of accelerating the ions to the required precipitation energies are dayside reconnection and KHIs along the magnetopause boundary. These processes may be linked through possible advection of ULF waves from noon to dusk, producing stronger nonlinear KHI waves along KH-unstable regions. We frame these observations with previous key studies analyzing the X-ray aurora; models suggesting plausible drivers and ULF wave activity in the jovian magnetosphere, providing the foundations for future studies.

We hope that the work presented here helps narrow down the list of possible drivers that produce the X-ray auroral emissions using a consistent definition and numerical threshold and sets the foundations for further exploration. The idea of the soft X-rays being confined to a single "hot spot" (i.e. produced by one driver) seems less likely from the results we show here. It is clear that in order to fully understand the driver and variability of the X-ray aurora, we need to apply these techniques to multiwavelength data (both in situ and remote sensing data such as XMM-Newton and the Hubble Space Telescope (HST)) to find any key correlations. With Juno's extended science mission taking the spacecraft through dusk-midnight sector, a similar statistical study can be carried out for the South pole with comparisons made between the poles. From there, we can then truly understand how the X-rays behave on a more planet-wide scale and the implications that has on the possible drivers as well as allowing us to fully understand the asymmetries we observe between North and South in X-rays and across many wavelengths.

\section{Acknowledgments}

D. M. W. is supported by the Science and Technoology Facilities Council (STFC) studentship ST/S505705/1. C. M. J.'s work at DIAS is supported by the Science Foundation Ireland (SFI) Grant 18/FRL/6199. C. K. L and S. C. M are also supported by SFI grant 18/FRL/6199. H. M. is supported by a Royal Society PhD studentship. W.R.D. was supported by a STFC research grant ST/S000240/1 to University College London (UCL). G. B.-R. acknowledges partial support from STFC Consolidated Grant ST/S000240/1 to UCL. This research has made use of data obtained from the Chandra Data Archive and Chandra Source Catalogue (https://cda.harvard.edu/chaser/). The data required 
to reproduce the results shown in this study are stored in the Zenodo repository at DOI: $10.5281 /$ zenodo. 4275744 .

\section{References}

Bhardwaj, A., \& Gladstone, G. R. (2000). Auroral emissions of the giant planets. Reviews of Geophysics, 38(3), 295-353. doi: 10.1029/1998RG000046

Bolton, S. J., Lunine, J., Stevenson, D., Connerney, J. E. P., Levin, S., Owen, T. C., ... Thorpe, R. (2017). The Juno Mission. Space Science Reviews, 213(1-4), 5-37. Retrieved from http://dx.doi.org/10.1007/s11214-017 -0429-6http://link. springer.com/10.1007/s11214-017-0429-6 doi: $10.1007 / \mathrm{s} 11214-017-0429-6$

Branduardi-Raymont, G., Bhardwaj, A., Elsner, R. F., Gladstone, G. R., Ramsay, G., Rodriguez, P., ... Cravens, T. E. (2007). Latest results on Jovian disk X-rays from XMM-Newton. Planetary and Space Science, 55(9), 1126-1134. doi: $10.1016 /$ j.pss.2006.11.017

Branduardi-Raymont, G., Elsner, R. F., Galand, M., Grodent, D., Cravens, T. E., Ford, P., ... Waite, J. H. (2008). Spectral morphology of the X-ray emission from Jupiter's aurorae. Journal of Geophysical Research: Space Physics, 113(2), 1-11. doi: 10.1029/2007JA012600

Bunce, E. J., Cowley, S. W., \& Yeoman, T. K. (2004). Jovian cusp processes: Implications for the polar aurora. Journal of Geophysical Research: Space Physics, 109(A9), 1-26. doi: 10.1029/2003JA010280

Clark, G., Mauk, B. H., Kollmann, P., Paranicas, C., Bagenal, F., Allen, R. C., ... Westlake, J. H. (2020). Heavy Ion Charge States in Jupiter's Polar Magnetosphere Inferred From Auroral Megavolt Electric Potentials. Journal of Geophysical Research: Space Physics, 125(9), 1-12. doi: 10.1029/2020ja028052

Connerney, J. E., Acuña, M. H., Ness, N. F., \& Satoh, T. (1998). New models of Jupiter's magnetic field constrained by the Io flux tube footprint. Journal of Geophysical Research: Space Physics, 103(A6), 11929-11939. doi: 10.1029/97ja03726

Connerney, J. E., Benn, M., Bjarno, J. B., Denver, T., Espley, J., Jorgensen, J. L., ... Smith, E. J. (2017). The Juno Magnetic Field Investigation. Space Science Reviews, 213(1-4), 39-138. Retrieved from http://dx.doi.org/10.1007/ s11214-017-0334-z doi: 10.1007/s11214-017-0334-z

Connerney, J. E., Kotsiaros, S., Oliversen, R. J., Espley, J. R., Joergensen, J. L., Joergensen, P. S., ... Levin, S. M. (2018). A New Model of Jupiter's Magnetic Field From Juno's First Nine Orbits. Geophysical Research Letters, 45(6), 2590-2596. doi: 10.1002/2018GL077312

Cravens, T. E., Howell, E., Waite, J. H., \& Gladstone, G. R. (1995). Auroral oxygen precipitation at Jupiter. Journal of Geophysical Research, 100(A9), 17153. doi: $10.1029 / 95 j a 00970$

Cravens, T. E., Waite, J. H., Gombosi, T. I., Lugaz, N., Gladstone, G. R., Mauk, B. H., \& MacDowall, R. J. (2003). Implications of Jovian X-ray emission for magnetosphere-ionosphere coupling. Journal of Geophysical Research: Space Physics, 108(A12), 1-12. doi: 10.1029/2003JA010050

Cutler, J. C., Dougherty, M. K., Lucek, E., \& Masters, A. (2011). Evidence of surface wave on the dusk flank of Saturn's magnetopause possibly caused by the Kelvin-Helmholtz instability. Journal of Geophysical Research: Space Physics, 116(10), 1-9. doi: 10.1029/2011JA016643

Delamere, P. A., \& Bagenal, F. (2010). Solar wind interaction with Jupiter's magnetosphere. Journal of Geophysical Research: Space Physics, 115(10), 1-20. doi: 10.1029/2010JA015347

Delamere, P. A., Wilson, R. J., Eriksson, S., \& Bagenal, F. (2013). Magnetic signatures of Kelvin-Helmholtz vortices on Saturn's magnetopause: Global 
survey. Journal of Geophysical Research: Space Physics, 118(1), 393-404. doi: 10.1029/2012JA018197

Desroche, M., Bagenal, F., Delamere, P. A., \& Erkaev, N. (2012). Conditions at the expanded Jovian magnetopause and implications for the solar wind interaction. Journal of Geophysical Research: Space Physics, 117(7), 1-18. doi: 10.1029/2012JA017621

Dungey, J. W. (1955). Electrodynamics of the Outer Atmosphere. In Physics of the ionosphere, report of the conference held at the cavendish laboratory (p. 229). Physics Society of London, London. Retrieved from https://ui.adsabs.harvard.edu/abs/1955phio.conf..229D

Dunn, W. R., Branduardi-Raymont, G., Carter-Cortez, V., Campbell, A., Elsner, R., Ness, J.-U., ... Achilleos, N. (2020b). Jupiter's Xray Emission During the 2007 Solar Minimum. Journal of Geophysical Research: Space Physics, 125(6), e2019JA027219. Retrieved from https://onlinelibrary.wiley.com/doi/ abs/10.1029/2019JA027219 doi: 10.1029/2019JA027219

Dunn, W. R., Branduardi-Raymont, G., Elsner, R. F., Vogt, M. F., Lamy, L., Ford, P. G., .. Jasinski, J. M. (2016). The impact of an ICME on the Jovian X-ray aurora. Journal of Geophysical Research A: Space Physics, 121(3), 2274-2307. doi: $10.1002 / 2015 J A 021888$

Dunn, W. R., Branduardi-Raymont, G., Ray, L. C., Jackman, C. M., Kraft, R. P., Elsner, R. F., ... Coates, A. J. (2017). The independent pulsations of Jupiter's northern and southern X-ray auroras. Nature Astronomy, 1(11), 758-764. Retrieved from http://dx.doi.org/10.1038/s41550-017-0262-6 doi: 10.1038/s41550-017-0262-6

Dunn, W. R., Gray, R., Wibisono, A. D., Lamy, L., Louis, C., Badman, S. V., ... Kraft, R. (2020a). Comparisons Between Jupiter's Xray, UV and Radio Emissions and InSitu Solar Wind Measurements During 2007. Journal of Geophysical Research: Space Physics, 125(6), e2019JA027222. Retrieved from https://onlinelibrary.wiley.com/doi/abs/10.1029/2019JA027222 doi: 10.1029/2019JA027222

Ebert, R. W., Allegrini, F., Bagenal, F., Bolton, S. J., Connerney, J. E., Clark, G., ... Wilson, R. J. (2017). Accelerated flows at Jupiter's magnetopause: Evidence for magnetic reconnection along the dawn flank. Geophysical Research Letters, 44 (10). doi: 10.1002/2016GL072187

Efron, B., \& Stein, C. (1981). The Jackknife Estimate of Variance. The Annals of Statistics, 9(3), 586-596. Retrieved from http://projecteuclid.org/ euclid.aos/1176345976http://projecteuclid.org/euclid.aos/ 1176348654http://projecteuclid.org/euclid.aos/1176345462 doi: 10.1214/aos/1176345462

Elsner, R. F., Lugaz, N., Waite, J. H., Cravens, T. E., Gladstone, G. R., Ford, P., ... Majeed, T. (2005). Simultaneous Chandra X ray Hubble Space Telescope ultraviolet, and Ulysses radio observations of Jupiter's aurora. Journal of Geophysical Research: Space Physics, 110(A1), 1-16. doi: 10.1029/2004JA010717

Farrugia, C. J., Gratton, F. T., Bender, L., Biernat, H. K., Erkaev, N. V., Quinn, J. M., ... Dennisenko, V. (1998). Charts of joint Kelvin-Helmholtz and Rayleigh-Taylor instabilites at the dayside magnetopause for strongly northward interplanetary magnetic field. Journal of Geophysical Research: Space Physics, 103(A4), 6703-6727. doi: 10.1029/97ja03248

Foullon, C., Farrugia, C. J., Fazakerley, A. N., Owen, C. J., Gratton, F. T., \& Torbert, R. B. (2008). Evolution of Kelvin-Helmholtz activity on the dusk flank magnetopause. Journal of Geophysical Research: Space Physics, 113(11), 1-22. doi: $10.1029 / 2008 J A 013175$

Gladstone, G. R., Waite, J. H., Grodent, D., Lewis, W. S., Crary, F. J., Elsner, R. F., ... Cravens, T. E. (2002). A pulsating auroral X-ray hot spot on Jupiter. $\quad$ Nature, 415(6875), 1000-1003. Retrieved from http:// 
www.nature.com/articles/4151000a doi: 10.1038/4151000a

Grodent, D., Bonfond, B., Gérard, J. C., Radioti, A., Gustin, J., Clarke, J. T., ... Connerney, J. E. (2008). Auroral evidence of a localized magnetic anomaly in Jupiter's northern hemisphere. Journal of Geophysical Research: Space Physics, 113(9), 1-10. doi: 10.1029/2008JA013185

Guo, R. L., Yao, Z. H., Sergis, N., Wei, Y., Mitchell, D., Roussos, E., ... Wan, W. X. (2018). Reconnection Acceleration in Saturn's Dayside Magnetodisk: A Multicase Study with Cassini . The Astrophysical Journal, 868(2), L23. Retrieved from http://dx.doi.org/10.3847/2041-8213/aaedab doi: 10.3847/2041-8213/aaedab

Hasegawa, H., Fujimoto, M., Phan, T.-D., Rème, H., Balogh, A., Dunlop, M. W., .. . Tandokoro, R. (2004). Transport of solar wind into Earth's magnetosphere through rolled-up Kelvin-Helmholtz vortices. Nature, 430(7001), 755-758. doi: 10.1038 /nature02799

Hess, S. L., Bonfond, B., Zarka, P., \& Grodent, D. (2011). Model of the Jovian magnetic field topology constrained by the Io auroral emissions. Journal of Geophysical Research: Space Physics, 116(5), 1-19. doi: 10.1029/2010JA016262

Houston, S. J., Cravens, T. E., Schultz, D. R., Gharibnejad, H., Dunn, W. R., Haggerty, D. K., .. Ozak, N. (2020). Jovian Auroral Ion Precipitation: XRay Production From Oxygen and Sulfur Precipitation. Journal of Geophysical Research: Space Physics, 125(2), 2019JA027007. Retrieved from https://onlinelibrary.wiley.com/doi/10.1029/2019JA027007 doi: 10.1029/2019JA027007

Huddleston, D. E., Russell, C. T., Le, G., \& Szabo, A. (1997). Magnetopause structure and the role of reconnection at the outer planets. Journal of Geophysical Research A: Space Physics, 102(A11), 24289-24302. doi: 10.1029/97JA02416

Hui, Y., Schultz, D. R., Kharchenko, V. A., Bhardwaj, A., Branduardi-Raymont, G., Stancil, P. C., ... Dalgarno, A. (2010). Comparative analysis and variability of the Jovian X-ray spectra detected by the Chandra and XMM-Newton observatories. Journal of Geophysical Research: Space Physics, 115(A7), 1-19. doi: 10.1029/2009ja014854

Jackman, C. M., Knigge, C., Altamirano, D., Gladstone, R., Dunn, W., Elsner, R., ... Ford, P. (2018). Assessing Quasi-Periodicities in Jovian X-Ray Emissions: Techniques and Heritage Survey. Journal of Geophysical Research: Space Physics, 123(11), 9204-9221. doi: 10.1029/2018JA025490

Jansen, F., Lumb, D., Altieri, B., Clavel, J., Ehle, M., Erd, C., .. V Vacanti, G. (2001). XMM-Newton spacecraft and operations. Astronomy and Astrophysics, 365, L1-L6. doi: 10.1051/0004-6361

Joy, S. P., Kivelson, M. G., Walker, R. J., Khurana, K. K., Russell, C. T., \& Ogino, T. (2002). Probabilistic models of the Jovian magnetopause and bow shock locations. Journal of Geophysical Research: Space Physics, 107(A10), 1-17. doi: 10.1029/2001JA009146

Khurana, K. K., \& Kivelson, M. G. (1989). Ultralow frequency MHD waves in Jupiter's middle magnetosphere. Journal of Geophysical Research, 94(A5), 5241. doi: 10.1029/ja094ia05p05241

Kimura, T., Kraft, R. P., Elsner, R. F., Branduardi-Raymont, G., Gladstone, G. R., Tao, C., ... Murray, S. S. (2016). Jupiter's X-ray and EUV auroras monitored by Chandra, XMM-Newton, and Hisaki satellite. Journal of Geophysical Research A: Space Physics, 121(3), 2308-2320. doi: 10.1002/2015JA021893

Kohlhase, C., \& Penzo, P. (1977). Voyager mission description. Space Science Reviews, 21(2). Retrieved from http://link.springer.com/10.1007/ BF00200846 doi: 10.1007/BF00200846

Krimigis, S. M., Mitchell, D. G., Hamilton, D. C., Dandouras, J., Armstrong, T. P., Bolton, S. J., ... Williams, D. J. (2002). A nebula of gases from Io surrounding Jupiter. Nature, 415(6875), 994-996. doi: 10.1038/415994a 
Lysak, R. L., \& Song, Y. (2020). Field Line Resonances in Jupiter's Magnetosphere. Geophysical Research Letters, 47(18). doi: 10.1029/2020GL089473

Ma, X., Stauffer, B., Delamere, P. A., \& Otto, A. (2015). Asymmetric KelvinHelmholtz propagation at Saturn's dayside magnetopause. Journal of Geophysical Research: Space Physics, 120(3), 1867-1875. Retrieved from http://doi .wiley.com/10.1002/2014JA020746 doi: 10.1002/2014JA020746

MacDowall, R. J., Kaiser, M. L., Desch, M. D., Farrell, W. M., Hess, R. A., \& Stone, R. G. (1993). Quasiperiodic Jovian Radio bursts: observations from the Ulysses 1. MacDowall, R. J. et al. Quasiperiodic Jovian Radio bursts: observations from the Ulysses Radio and Plasma Wave Experiment. Planet. Space Sci. 41, 10591072 (1993).Radio and Plasma Wave Ex. Planetary and Space Science, 41(11-12), 1059-1072. doi: 10.1016/0032-0633(93)90109-F

Mann, I. R., Voronkov, I., Dunlop, M., Donovan, E., Yeoman, T. K., Milling, D. K., ... Opgenoorth, H. J. (2002). Coordinated ground-based and Cluster observations of large amplitude global magnetospheric oscillations during a fast solar wind speed interval. Annales Geophysicae, 20(4), 405-426. doi: 10.5194/angeo-20-405-2002

Manners, H., \& Masters, A. (2020). The Global Distribution of UltraLowFrequency Waves in Jupiter's Magnetosphere. Journal of Geophysical Research: Space Physics. Retrieved from https://doi.org/10.1002/essoar.10503411 .1https://onlinelibrary.wiley.com/doi/10.1029/2020JA028345 doi: 10.1029/2020JA028345

Manners, H., Masters, A., \& Yates, J. N. (2018). Standing Alfvén Waves in Jupiter's Magnetosphere as a Source of $~ 10$ - to 60-Min Quasiperiodic Pulsations. Geophysical Research Letters, 45(17), 8746-8754. Retrieved from http:// doi.wiley.com/10.1029/2018GL078891 doi: 10.1029/2018GL078891

Masters, A. (2018). A More Viscous-Like Solar Wind Interaction With All the Giant Planets. Geophysical Research Letters, 45(15), 7320-7329. doi: 10.1029/ 2018GL078416

Masters, A., Achilleos, N., Bertucci, C., Dougherty, M. K., Kanani, S. J., Arridge, C. S., ... Coates, A. J. (2009). Surface waves on Saturn's dawn flank magnetopause driven by the Kelvin-Helmholtz instability. Planetary and Space Science, 57(14-15), 1769-1778. Retrieved from http://dx.doi.org/10.1016/ j.pss. 2009.02.010 doi: 10.1016/j.pss.2009.02.010

Masters, A., Eastwood, J. P., Swisdak, M., Thomsen, M. F., Russell, C. T., Sergis, N., ... Krimigis, S. M. (2012). The importance of plasma $\beta$ conditions for magnetic reconnection at Saturn's magnetopause. Geophysical Research Letters, 39(8), 1-6. doi: 10.1029/2012GL051372

Mauk, B. H., Haggerty, D. K., Jaskulek, S. E., Schlemm, C. E., Brown, L. E., Cooper, S. A., ... Stokes, M. R. (2017). The Jupiter Energetic Particle Detector Instrument (JEDI) Investigation for the Juno Mission. Space Science Reviews, 213(1-4), 289-346. doi: 10.1007/s11214-013-0025-3

McComas, D. J., Alexander, N., Allegrini, F., Bagenal, F., Beebe, C., Clark, G., .. . White, D. (2017). The Jovian Auroral Distributions Experiment (JADE) on the Juno Mission to Jupiter. Space Science Reviews, 213(1-4), 547-643. Retrieved from http://dx.doi.org/10.1007/s11214-013-9990-9 doi: 10.1007/s11214-013-9990-9

Moore, K. M., Yadav, R. K., Kulowski, L., Cao, H., Bloxham, J., Connerney, J. E., ... Levin, S. M. (2018). A complex dynamo inferred from the hemispheric dichotomy of Jupiter's magnetic field. Nature, 561(7721), 76-78. Retrieved from http://dx.doi.org/10.1038/s41586-018-0468-5 doi: 10.1038/s41586-018-0468-5

Northrop, T. G., Goertz, C. K., \& Thomsen, M. F. (1974). The magnetosphere of Jupiter as observed with Pioneer 10: 2. Nonrigid rotation of the magnetodisc. Journal of Geophysical Research, 79(25), 3579-3582. doi: 


\subsection{9/ja079i025p03579}

Nykyri, K., \& Otto, A. (2001). Plasma transport at the magnetospheric boundary due to reconnection in Kelvin-Helmholtz vortices. Geophysical Research Letters, 28(18), 3565-3568. Retrieved from http://doi.wiley.com/10.1029/ 2001GL013239 doi: 10.1029/2001GL013239

Ozak, N., Cravens, T. E., \& Schultz, D. R. (2013). Auroral ion precipitation at Jupiter: Predictions for Juno. Geophysical Research Letters, 40(16), 41444148. doi: $10.1002 /$ grl.50812

Ozak, N., Schultz, D. R., Cravens, T. E., Kharchenko, V., \& Hui, Y. W. (2010). Auroral X-ray emission at Jupiter: Depth effects. Journal of Geophysical Research: Space Physics, 115(11), 1-13. doi: 10.1029/2010JA015635

Quenouille, M. H. (1949). Problems in Plane Sampling. The Annals of Mathematical Statistics, 20(3), 355-375. Retrieved from http://projecteuclid.org/ euclid.aoms/1177706647http://projecteuclid.org/euclid.aoms/ 1177729989 doi: $10.1214 /$ aoms/1177729989

Quenouille, M. H. (1956). Notes on Bias in Estimation. Biometrika, 43(3/4), 353. doi: $10.2307 / 2332914$

Rae, I. J., Donovan, E. F., Mann, I. R., Fenrich, F. R., Watt, C. E., Milling, D. K., ... Balogh, A. (2005). Evolution and characteristics of global Pc5 ULF waves during a high solar wind speed interval. Journal of Geophysical Research: Space Physics, 110(A12), 1-16. doi: 10.1029/2005JA011007

Russell, C. T. (Ed.). (1992). The Galileo Mission. Dordrecht: Springer Netherlands. Retrieved from http://link.springer.com/10.1007/978-94-011-2512-3 doi: 10.1007/978-94-011-2512-3

Sandel, B. R., Smith, E. J., Davis, L., Jones, D. E., Coleman, P. J., Colburn, D. S., .. Sonett, C. P. (1975). Jupiter's Magnetic Field. Magnetosphere, and Interaction with the Solar Wind: Pioneer 11. Science, 188(4187), 451455. Retrieved from https://www.sciencemag.org/lookup/doi/10.1126/ science.188.4187.451 doi: 10.1126/science.188.4187.451

Vogt, M. F., Bunce, E. J., Kivelson, M. G., Khurana, K. K., Walker, R. J., Radioti, A., ... Grodent, D. (2015). Magnetosphere-ionosphere mapping at Jupiter: Quantifying the effects of using different internal field models. Journal of Geophysical Research: Space Physics, 120(4), 2584-2599. doi: 10.1002/2014JA020729

Vogt, M. F., Gyalay, S., Kronberg, E. A., Bunce, E. J., Kurth, W. S., Zieger, B., \& Tao, C. (2019). Solar Wind Interaction With Jupiter's Magnetosphere: A Statistical Study of Galileo In Situ Data and Modeled Upstream Solar Wind Conditions. Journal of Geophysical Research: Space Physics, 1-30. doi: 10.1029/2019JA026950

Vogt, M. F., Kivelson, M. G., Khurana, K. K., Walker, R. J., Bonfond, B., Grodent, D., \& Radioti, A. (2011). Improved mapping of Jupiter's auroral features to magnetospheric sources. Journal of Geophysical Research: Space Physics, 116 (3). doi: 10.1029/2010JA016148

Weigt, D. M., Jackman, C. M., Dunn, W. R., Gladstone, G. R., Vogt, M. F., Wibisono, A. D., ... Kraft, R. P. (2020). Chandra Observations of Jupiter's Xray Auroral Emission During Juno Apojove $2017 . \quad$ Journal of Geophysical Research: Planets, 125(4), e2019JE006262. Retrieved from https://onlinelibrary.wiley.com/doi/abs/10.1029/2019JE006262 doi: 10.1029/2019JE006262

Weisskopf, M. C., Tananbaum, H. D., Van Speybroeck, L. P., \& O’Dell, S. L. (2000). Chandra X-ray Observatory (CXO): overview. X-Ray Optics, Instruments, and Missions III, 4012(July 2000), 2-16. doi: 10.1117/12.391545

Wenzel, K. P., Marsden, R. G., Page, D. E., \& Smith, E. J. (1992). The ULYSSES Mission. Astronomy and Astrophysics Supplement, 92(2), 207.

Wibisono, A. D., Branduardi-Raymont, G., Dunn, W. R., Coates, A. J., Weigt, 
D. M., Jackman, C. M., ... Fleming, D. (2020). Temporal and Spectral Studies by XMM-Newton of Jupiter's X-ray Auroras During a Compression Event. Journal of Geophysical Research: Space Physics, 125(5), e2019JA027676.

Retrieved from http://doi.wiley.com/10.1029/2019JA027676 doi: 10.1029/2019JA027676

Wilson, R. J., \& Dougherty, M. K. (2000). Evidence provided by galileo of ultra low frequency waves within Jupiter's middle magnetosphere. Geophysical Research Letters, 27(6), 835-838. doi: 10.1029/1999GL010750

Zhang, B., Delamere, P. A., Ma, X., Burkholder, B., Wiltberger, M., Lyon, J. G., ... Sorathia, K. A. (2018). Asymmetric Kelvin-Helmholtz Instability at Jupiter's Magnetopause Boundary: Implications for Corotation-Dominated Systems.

Geophysical Research Letters, 45(1), 56-63. doi: 10.1002/2017GL076315 